
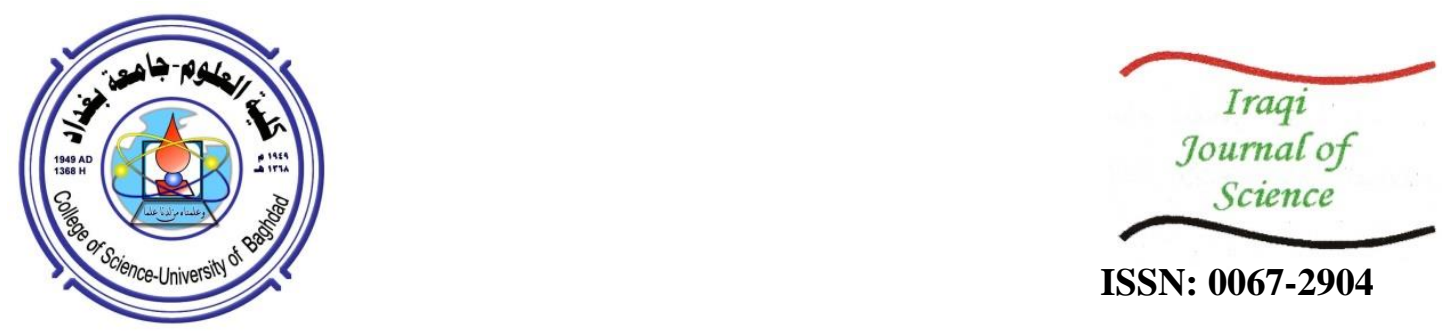

\title{
Topological Structure of Nested Chain Abacus
}

\author{
Eman F. Mohommed \\ Department of Mathematics, College of Education, Mustansiriyah University, Baghdad, Iraq \\ Received: 11/12/ $2019 \quad$ Accepted: 15/3/2020

\begin{abstract}
This paper focuses on developing a strategy to represent the $n$-connected ominoes using an abacus. We use the idea of $n$-connected ominoes with respect to a frame in modelling nested chain abacus. Then, we formulate and prove the unique connected partition for any $n$-connected ominoes. Next, the topological structure of nested chain abacus is presented.
\end{abstract}

Keywords: $n$-connected ominoes, Abacus, Nested Chain, Connected Partition
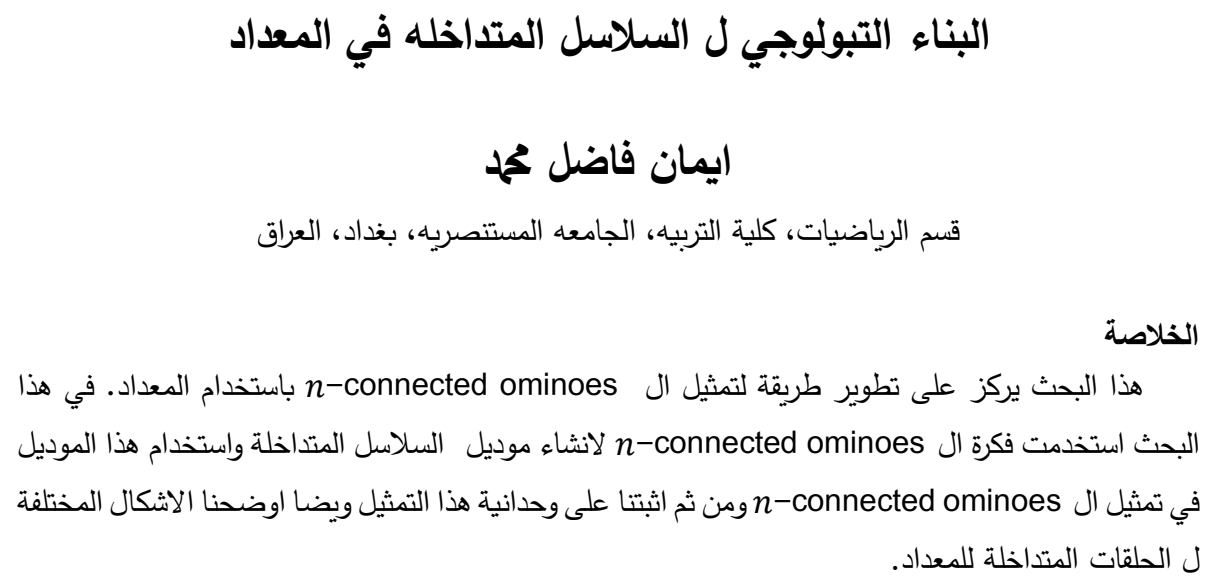

\section{Introduction}

Abacus model, first introduced by D.G. James (1978), was used to give a new representation of any partition $\mu$ of a positive integer $t$ by using a sequence of positive integer numbers called beta numbers. The formal definition of James abacus model states that it is an abacus with $e$ columns numbered $0,1, \ldots$, $e-1$ from left to right and a configuration of beta numbers which are present across them. On column $k$, the positions are labelled $k, k+e, k+2 e, \ldots$ from top to down. The abacus model was established to construct fundamental facts of partitions [1-3]. Futhermore, several identities such as Schur polynomials and Littlewood Richardson rule [4], lecture hall [5], and nested chain [6] were employing the idea of abacus model. However, the existing abacus model involves one or more empty columns or empty rows with each partition has $n-1$ representation. Thus, in this paper we construct an abacus with a nested chain that is called nested chain abacus. The new abacus proposes a new partition on $n$-connected ominoes to design the connected partition. Hence, this paper aims to develop the structure of the nested chain abacus and derive properties related to the nested chain. The fundamental concept about the establishment of the nested chain abacus is then discussed in Section 2.

\section{Definitions and Terminologies}

This section provides definitions needed to develop nested chain abacus. 


\section{Definition 2.1}

The $n$-connected ominoes is a plane geometric figure formed by one or more ominoes (equal squares), such that there exists a path from one ominoes to another for any pair of ominoes which are internally connected and have holes.

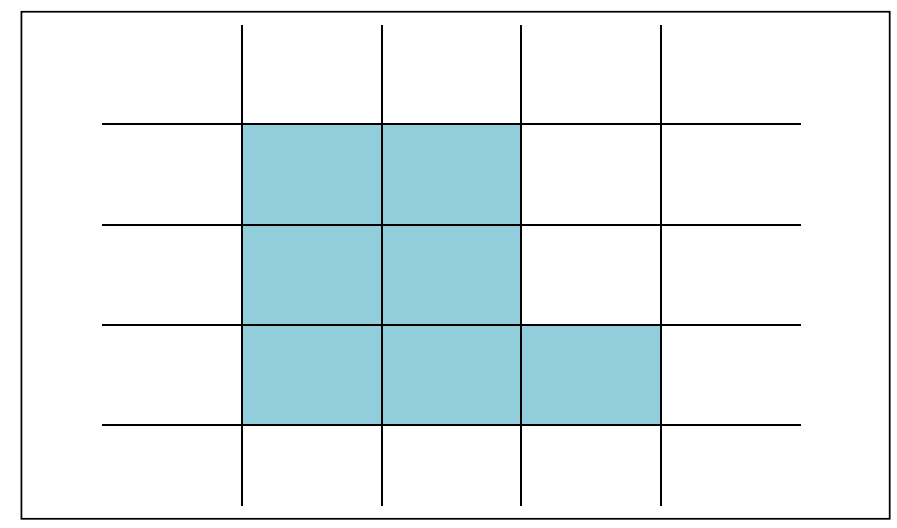

Figure 1-A 7-connected ominoes.

Next, we define the graphical form of $n$-connected ominoes with respect to a minimal frame, which enables us to define ominoe positions and empty ominoe positions in terms of the rows and columns of the minimal frame.

\section{Definition 2.2}

A minimal frame with $r$ rows and $e$ columns for any $n$-connected ominoes is such that there is at least one ominoes in each column and each row where $n \leqslant e r$.

Consider Figure- 1 where the minimal frame of 7-connected ominoes is as illustrated in Figure-2.

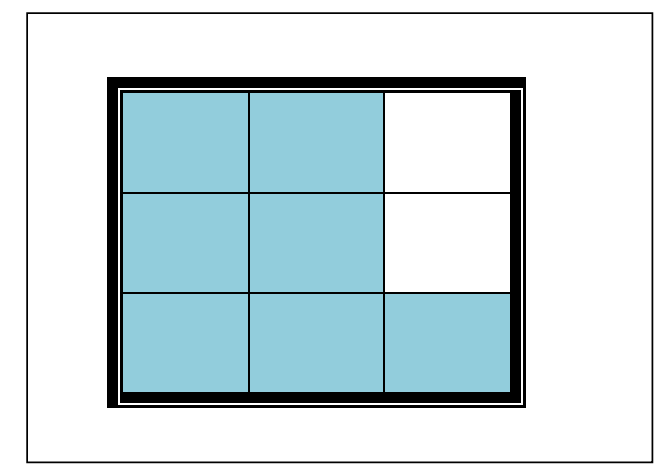

Figure 2-7-connected ominoes in a minimal frame.

\section{Definition 3}

Any $n$-connected ominoes in a minimal frame of $r \times e$ can be represented in a nested chain abacus with $n$ beads where each bead position $w_{k}$ is obtained using the function $f(m, j): Z \times Z \rightarrow Z$, such that, if location $(m, j)$ in the minimal frame contains an ominoes, then

$$
w_{k}=f(m, j)=(m-1) e+(j-1)
$$

for $k=1,2, \ldots, n$ where $m$ and $j$ refer to the number of the rows and columns of the minimal frame, respectively. A nested chain abacus is an abacus with $e$ columns, $r$ rows, $c$ chains and $n$ connected bead positions, which satisfy the following conditions:

1. The columns are labelled from left to right as $0,1, \ldots, e-1$.

2. The rows are labelled from up to down as $0,1, \ldots, r-1$.

3. The placement of a bead on the new abacus in each position is given by $f$.

4. The bead locations are labelled with numbers $0,1, \ldots, e r-1$ across the rows from left to right, beginning from the number 0 in the top-leftmost location until the number er-1 in the bottom-rightmost location.

5. The chains are labelled $1,2, \ldots, c$, and if $i=1$ the chain is called outer chain while if $i>1$ the 
chains are called inner chains.

6. Each column and row have at lest one bead position.

Table-1 shows the placement of position numbers on the nested chain abacus with re positions.

Table 1-Nested chain abacus with re bead positions.

\begin{tabular}{|c|c|c|c|c|}
\hline colu.1 & colu. 2 & colu. 3 & $\ldots$ & colu. $\mathrm{e}-1$ \\
\hline & 1 & 2 & $\ldots$ & $\mathrm{e}-1$ \\
\hline $\mathrm{e}$ & $2 \mathrm{e}+1$ & $2 \mathrm{e}+2$ & $\ldots$ & $3 \mathrm{e}-1$ \\
\hline. &. &. & $\ldots$ &. \\
\hline. &. &. & $\ldots$ &. \\
\hline. &. &. & $\ldots$ &. \\
\hline $\mathrm{e}(\mathrm{r}-2)$ & $\mathrm{e}(\mathrm{r}-2)+1$ & $\mathrm{e}(\mathrm{r}-2)+2$ & $\ldots$ & $\mathrm{e}(\mathrm{r}-1)-1$ \\
\hline $\mathrm{e}(\mathrm{r}-1)$ & $\mathrm{e}(\mathrm{r}-1)+1$ & $\mathrm{e}(\mathrm{r}-1)+2$ & $\ldots$ & $\mathrm{re}-1$ \\
\hline
\end{tabular}

Every bead position will be represented by a circle $(\bigcirc)$ (Figure-2), while the empty bead position will be represented by a dashed circle.

A connected partition $\mu^{(e, r)}$ is a sequence of non-increasing positive integer numbers $\left(\mu_{1}^{\tau_{1}}, \mu_{2}^{\tau_{2}}, \ldots, \mu_{b}^{\tau_{b}}\right)$, such that $\mu_{b^{\prime}}$ represents connected bead positions and $\sum_{b^{\prime}=1}^{b} \tau_{b^{\prime}}=n$ where $n$ is the number of parts in the connected partition. Next, we will construct an algorithm for the connectedness of bead positions.

\section{Nested chain abacus}

A new representation of $n$-connected ominoes, called nested chain abacus, has been constructed. We use $n=7$ to explain the algorithm for nested chain abacus.

\section{Step 1: Establishing a graphical form of $\boldsymbol{n}$-connected ominoes}

Consider Figure-1(a) for 7-connected ominoes and 1(b) for the 7-connected ominoes in a minimal frame.
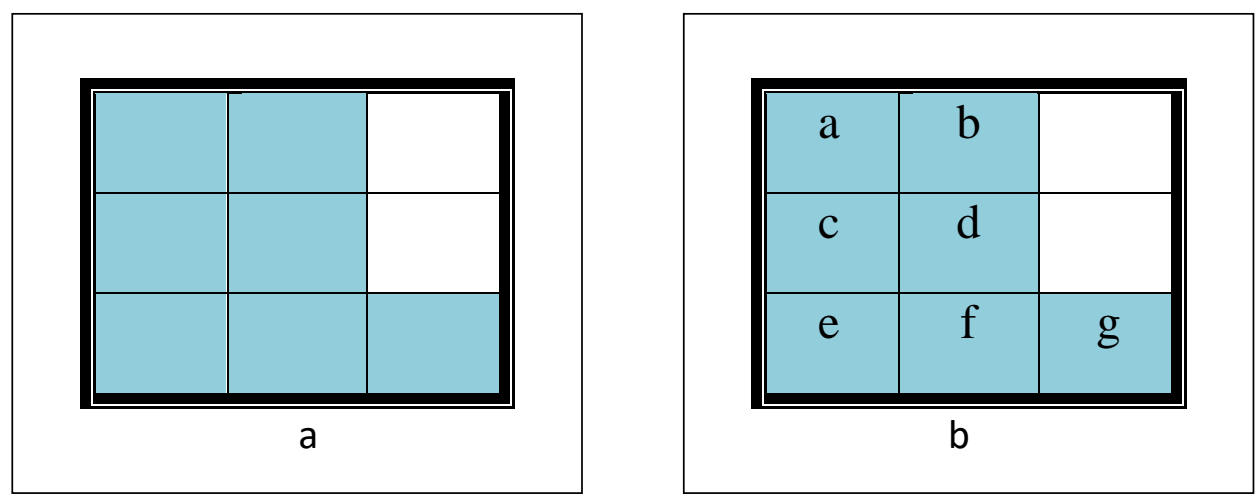

Figure 1-(a) 7-connected ominoes and (b) 7-connected ominoes in a minimal frame of 3 rows and 3 columns.

\section{Step 2: Creating a direction of $\boldsymbol{n}$-connected ominoes w.r.t minimal frame}

In this step, we created a direction to obtain nested chain abacus with respect to the minimal frame. We begin with the top-leftmost positions, from left to right, and from top to bottom in the minimal frame. Consider Figure- 1.b where we begin from bead position A which is located in row 1 and column 1 , then bead position B in row 1 and column 2 . The third, fourth,... positions for the minimal frame for 7-connected ominoes are substituted with the remaining positions C, D, E, F, G, respectively. Subsequently, we can also observe that there are two empty positions: The first empty position is in row 1 and column 3 , and the second in row 2 and column 3 .

\section{Step 3: Creating $n$-connected bead positions}

In this step, we followed the Definition 2 in creating bead positions on the nested chain abacus.

Consider the 7-connected ominoes in Figure-1.b, to find $w_{k}$ for $k=1$, in which we inspected the location in row 1 and column 1 . Since this location contains omino A, we calculate $w_{1}$ by applying the function $f$ where $\mathrm{m}=1$ and $\mathrm{j}=1$. Then $w_{1}=f(1,1)=(1-1) 3+(1-1)=0$. After that, we 
increment $k$ by 1 so that the next application of the function $f$ would yield $w_{2}$ where $w_{2}=f(1,2)=$ $(1-1) 3+(2-1)=1$.

The inspection process is continued in the same manner and subsequently function $f$ would only be applied accordingly to obtain $w_{3}=3, w_{4}=4, w_{5}=6, w_{6}=7$ and $w_{7}=8$. Consider Definition 2 in which the positions of the 7 beads in the nested chain abacus are as shown in Figure-2.
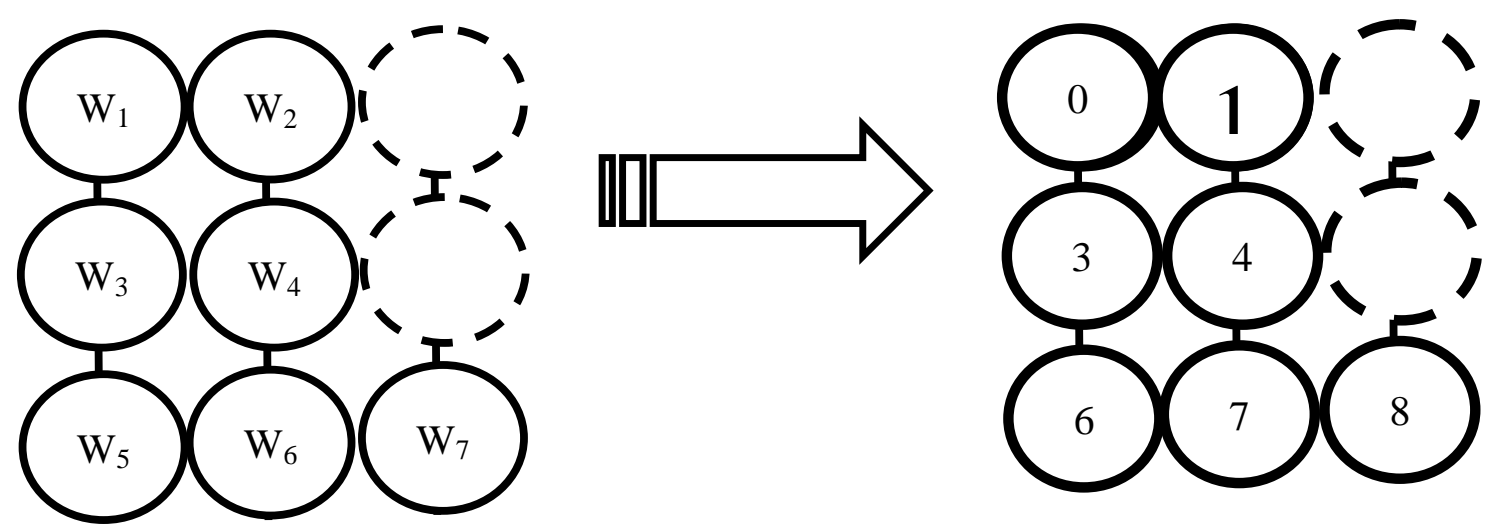

Figure 2-(a) The bead positions $w_{1}, w_{2}, \ldots, w_{7}$ in the nested chain abacus (b) Nested chain abacus with 7-beads.

\section{Step 4: Developing a connected partition of nested chain abacus}

Using the $w_{k}$ 's obtained from step 3 we produce a partition called the connected partition which represents the nested chain abacus for $n$-connected ominoes with $e$ columns and $r$ rows for $k=$ $1,2, \ldots, n$. The transformation process of the $w_{k}$ 's into the connected partition $\mu^{(e, r)}$ is as follows:

$\mu_{n}=w_{1}, \mu_{n-g}=w_{\mathrm{g}+1}-w_{\mathrm{g}}+\mu_{n-g+1}-1$, where $1 \leq g \leq n-1$

Then $\mu^{(e, r)}=\left(\mu_{1}^{t_{1}}, \mu_{2}^{t_{2}}, \ldots, \mu_{b}^{t_{b}}\right)$ is a connected position with $e$ columns and $r$ rows, where $\mu_{1} \geqslant \mu_{2} \geqslant$ $\cdots \geqslant \mu_{b}$ and $\sum_{b^{\prime}=1}^{b} t_{b^{\prime}}=n$.

Consider Figure-2, where we illustrates the process of finding connected partition $\mu^{(3,3)}$ for 7 -connected ominoes. Since $\left\{w_{1}, w_{2}, w_{3}, w_{4}, w_{5}, w_{6}, w_{7}\right\}=\{0,1,3,4,6,7,8\}$ then, $\mu^{(3,3)}=\left(2^{3}, 1^{2}, 0^{2}\right)$.

Based on the algorithm discussed earlier, we present a unique expression for $n$-connected ominoes, as shown in the Theorem 1. To prove it, we need Proposition 1 and Lemma 1.

Proposition 1: Let $f: Z \times Z \rightarrow Z$ be defined by $f(m, j)=e(m-1)+(j-1)$. $f$ is injections where $e$ and $r$ are positive integers for $1 \leqslant m \leqslant r$ and $1 \leqslant j \leqslant e$.

Proof. For every $(m, j)$ and $\left(m^{\prime}, j^{\prime}\right)$ where $m, j, m^{\prime}$ and $j^{\prime}$ are positive integers for $1 \leqslant m \leqslant r$ and $1 \leqslant j \leqslant e$. Suppose that $f(m, j)=f\left(m^{\prime}, j^{\prime}\right)$. If $m=m^{\prime}$ or $j=j^{\prime}$ then $(m, j)=\left(m^{\prime}, j^{\prime}\right)$. If $m \neq m^{\prime}$ and $j \neq j^{\prime}$ then $e=\frac{j^{\prime}-j}{m-m^{\prime}}<j^{\prime}$. This is a contradiction, therefore $(m, j)=\left(m^{\prime}, j^{\prime}\right)$.

Lemma 1: For every nested chain abacus $\mathfrak{N}$ for $n$-connected ominoes with $S$ beads, $e$ columns, $r$ rows and $c$ chains. Then, there exists a unique connected partition representing $\mathfrak{N}$ where $S=$ $\left\{w_{1}, w_{2}, \ldots, w_{n}\right\}$.

Proof. Suppose that $\mu^{(e, r)}, \lambda^{(e, r)}$ are two connected partitions represented by $\mathfrak{N}$ with $S$ beads, $e$ columns and $r$ rows. Since $\mu^{(e, r)}=\left(\mu_{1}^{t_{1}}, \mu_{2}^{t_{2}}, \ldots, \mu_{b}^{t_{b}}\right)$ represents $\mathfrak{N}$ then,

$\mu_{b}=w_{1}, w_{2}=w_{1}+1, \ldots, w_{t_{1}}=w_{t_{1}-1}+1$.

$\mu_{b-1}=w_{t_{1}+1}, w_{t_{1}+2}=w_{t_{1}+1}+1, \ldots, w_{t_{2}}=w_{t_{2}-1}+1$.

$\mu_{1}=w_{t_{b-1}+1}, w_{t_{b-1}+2}=w_{t_{b-1}+1}+1, \ldots, w_{t_{b}}=w_{t_{b}-1}+1$.

Additionally, we assume that $\lambda^{(e, r)}=\left(\lambda_{1}^{t_{1}}, \lambda_{2}^{t_{2}}, \ldots, \lambda_{b}^{t_{b}}\right)$ represents $\mathfrak{N}$, therefore,

$\lambda_{b}=w_{1}, w_{2}=w_{1}+1, \ldots, w_{t_{1}}=w_{t_{1}-1}+1$.

$\lambda-1=w_{t_{1}+1}, w_{t_{1}+2}=w_{t_{1}+1}+1, \ldots, w_{t_{2}}=w_{t_{2}-1}+1$. 
$\lambda_{1}=w_{t_{b-1}+1}, w_{t_{b-1}+2}=w_{t_{b-1}+1}+1, \ldots, w_{t_{b}}=w_{t_{b}-1}+1$.

Thus $\mu^{(e, r)}=\left(\mu_{1}^{t_{1}}, \mu_{2}^{t_{2}}, \ldots, \mu_{b}^{t_{b}}\right)$ is a connected partition represented by the nested chain abacus with $e$ columns, $r$ rows and $S$ bead positions, such that $S=\left\{w_{1}, w_{2}, \ldots, w_{n}\right\}$. Meanwhile, $\left(\lambda_{1}^{t_{1}}, \lambda_{2}^{t_{2}}, \ldots, \lambda_{b}^{t_{b}}\right)$ is a connected partition representing the nested chain abacus with with $e$ columns, $r$ rows and $S$ of beads positions. Hence, $\mathfrak{N}$ is an associator with exactly one connected partition.

Theorem 1: For any form of $n$-connected ominoes, there exists a unique connected partition $\mu^{(e, r)}$ representing it with rows $(r)$ and columns $(e)$.

Proof. On the contrary, suppose that $\mathfrak{N}_{1}$ and $\mathfrak{N}_{2}$ are two nested chain abacus with $e$ columns, $r$ rows and $n$ beads representing one form of $n$-connected ominoes. Based on the algorithm in step 2 , there exist $f: Z \times Z \rightarrow Z$ such that $f(m, j)=e(m-1)+(j-1)$ where $e \geqslant j, e>0$ and $m j \leqslant e r$. Since $\mathfrak{N}_{1}$ represents a $n$-connected ominoes w.r.t minimal frame with $e$ columns and $r$ rows, so $f(m, j)=w_{k}$. Meanwhile, $\mathfrak{N}_{2}$ represents an $n$-connected ominoes w.r.t minimal frame with $e$ columns and $r$ rows, then $f(m, j)=w_{k}^{\prime}$ where $(m, j)$ is a location in the minimal frame containing an omino, $1 \leqslant m \leqslant r$ and $1 \leqslant j \leqslant e$ for $k=1,2, \ldots, n$. Based on Proposition $1, f$ is injections so $w_{k}=w_{k}^{\prime}$. Thus, any form of $n$-connected ominoes is represented by exactly one $\mathfrak{N}$. Based on Lemma 3 , there exists a unique connected partition represented by $\mathfrak{N}$, thus there exists a unique connected partition that represents $n$-connected ominoes.

Previously, there was no representation for every shape of $n$-connected ominoes. However, by using nested chain abacus, we can associate each of the four shapes of 4-connected ominoes with a connected partition using the nested chain abacus. The result of the representation is shown in Figure-3 where each shape is represents by a unique connected partition.

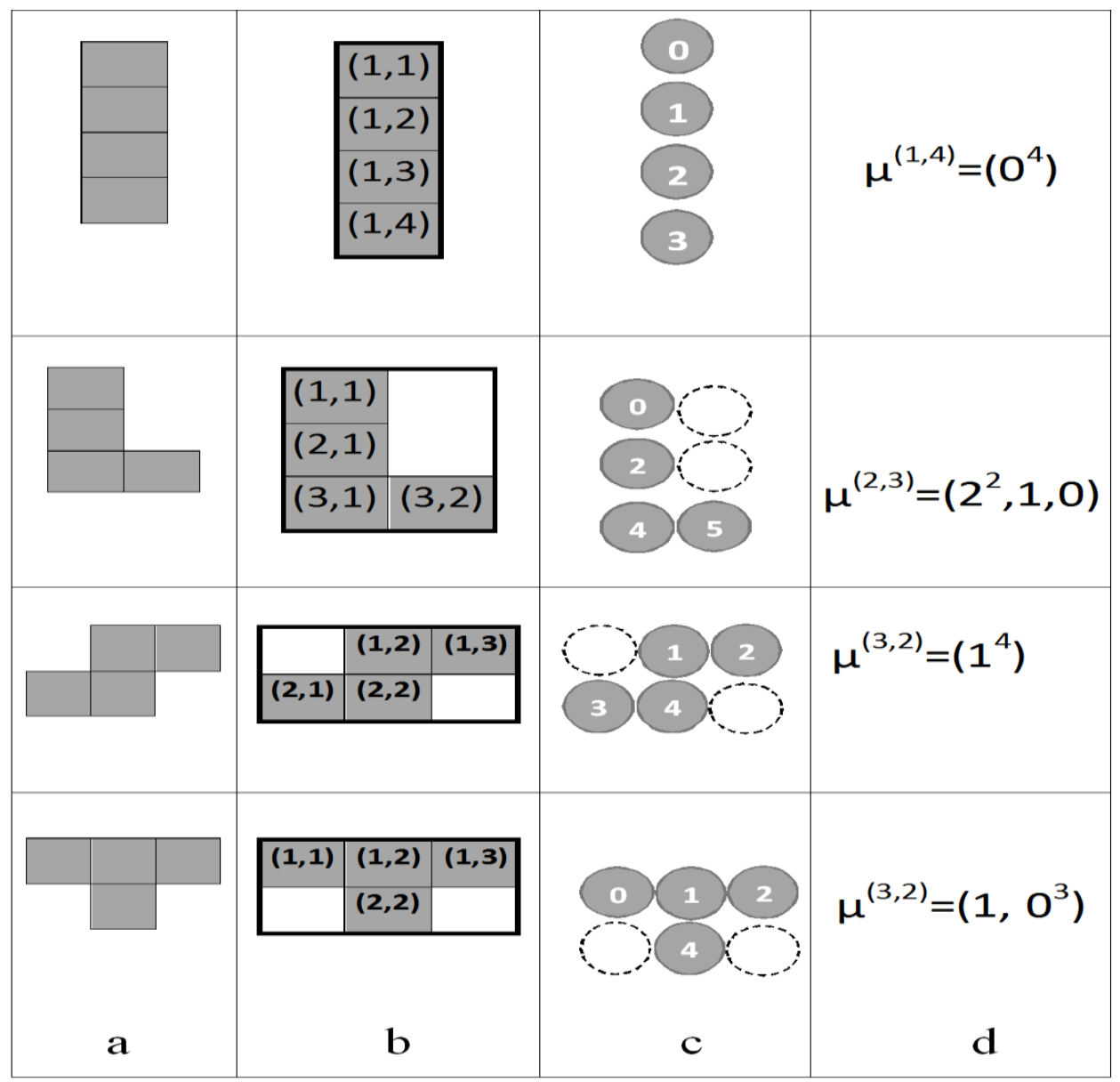

Figure 3-Representation of the 4 shapes of a family of tetromino. 


\section{Topological Structure of Nested Chain Abacus}

In this section, three design structures of the nested chain abacus are introduced; rectangular nested chain abacus, rectangle-path nested chain abacus, and square-singleton nested chain abacus. We begin by discussing the construction of rectangular nested chain abacus.

\subsection{Rectangular nested chain abacus}

The rectangular nested chain abacus consists of rectangular chains. Theorem 2 clarifies the construction of rectangular chains in nested chain abacus.

\section{Theorem 2}

Let there $r \times e$ matrix $A$ that represents bead positions and empty bead positions in the nested chain abacus with $e$ columns, $r$ rows and $c$ chains. Then,

1. A vertical rectangular chain is an arrangement of the bead positions and empty bead positions in a vertical rectangular format in the nested chain abacus, such that the element chain is

$$
\left\{a_{m i}, a_{m(e-i+1)}, a_{i j}, a_{(r-i+1) j} \mid i \leqslant m \leqslant(r-i+1), i \leqslant J \leqslant(e-i+1)\right\}
$$

where $e$ is an even number and $e \leqslant r$ for $1 \leqslant i \leqslant c$.

2. A horizontal rectangular chain is an arrangement of the bead positions and empty bead positions in a horizontal rectangular format in the nested chain abacus, such that the elements chain is

$$
\left\{a_{m i}, a_{m(e-i+1)}, a_{i j}, a_{(r-i+1) j} \mid i \leqslant m \leqslant(r-i+1), i \leqslant j \leqslant(e-i+1),\right\}
$$

where $r$ is an even number and $r<e$ for $1 \leqslant i \leqslant c$.

Proof.

1. A vertical rectangular chain starting from the $i$ th column will start at the $i^{\text {th }}$ row because rows 1 to $(i-1)$ will be covered by the vertical rectangular chains starting from rows $1,2, \ldots .,(i-1)$. After the starting point, we will come down along the same column so the row numbers will be changing and will come down till the $i$ th row from the end, so that it will be the $(r-i+1)^{\text {th }}$ row from the beginning. Now, in the $i$ th row, we go to the $(e-i+1)^{\text {th }}$ column and then we should cover the chain by coming down till the $(r-i+1)^{\mathrm{th}}$. So, two vertical columns have been covered. Now, from the starting point in the $i$ th row, we should cover up the chain on the right of it on that row till the $(e-i+1)^{\text {th }}$ column; so that the row will remain fixed and the columns will vary till we reach $(e-i+1)^{\text {th }}$ column in the $i$ th row and cover the chains in the $(r-i+1)^{\text {th }}$ row by keeping the row fixed and varying the columns from $i$ to $(e-i+1)$.

This is basically how it is done:

$a_{i i} \rightarrow a_{(r-i+1) i}$ then $a_{i\left(e \hat{a}^{\wedge} i+1\right)} \rightarrow a_{(r-i+1)(e-i+1)}$ to form the two vertical chains, and then to cover the rest; $a_{i(i+1)} \rightarrow a_{i(e-i)}$ and $a_{(r-i+1)(i+1)} \rightarrow a_{\left(r \hat{a}^{\wedge} i+1\right)\left(e \hat{a}^{\wedge} i\right)}$.

Thus the element chain will be:

$$
\left\{a_{m i}, a_{m(e-i+1)}, a_{i j}, a_{(r-i+1) j} \mid i \leqslant m \leqslant(r-i+1), i \leqslant J \leqslant(e-i+1)\right\}
$$

where $e$ is an even number and $e \leqslant r$ for $1 \leqslant i \leqslant c$.

2. Now, at the horizontal rectangular chain, the same situation arises so we cover the $i^{\text {th }}$ row chain starting from the $i$ th column and $i$ th row, then we cover up the chains in the $\left(r \hat{a}^{\wedge} 1+i\right)^{\text {th }}$ row and follow the same procedure to cover up the chains.

This is basically how it is done:

$a_{i i} \rightarrow a_{i(e-i+1)}$ then $a_{(r-i+1) i} \rightarrow a_{(r-i+1)(e-i+1)}$ to form the two horizontal chains, and then to cover the rest; $a_{(i+1) i} \rightarrow a_{\left(r \hat{a}^{\wedge} i\right) i}$ and $a_{(i+1)(e-i+1)} \rightarrow \rightarrow a_{(r-i)(e-i+1)}$.

and thus the element chain will be;

$$
\left\{a_{m i}, a_{m(e-i+1)}, a_{i j}, a_{(r-i+1) j} \mid i \leqslant m \leqslant(r-i+1), i \leqslant J \leqslant(e-i+1)\right\}
$$

where $r$ is an even number and $r<e$ for $1 \leqslant i \leqslant c$.

\subsection{Rectangle-path nested chain abacus}

The rectangle-path nested chain abacus consists of rectangular chains and a one path chain. Theorem 3 clarifies the construction of rectangle-path chain in nested chain abacus.

\section{Theorem 3}

Let the $r \times e$ matrix A represents bead positions and empty bead positions in the nested chain abacus with $e$ columns and $r$ rows. Then,

1. A vertical-path chain is an arrangement of some bead positions and empty bead positions in column $\frac{e+1}{2}$ in the nested chain abacus, such that the elements in the chain are 


$$
\frac{e+1}{2}=\left\{a_{m\left(\frac{e+1}{2}\right)}: \frac{e-1}{2} \leqslant m \leqslant \frac{2 r-e+3}{2}\right\}
$$

where $e<r, e$ is odd and $c$ is a positive integer.

2. A horizontal-path chain is an arrangement of some bead positions and empty bead positions in the row $\frac{r+1}{2}$ in the nested chain abacus, such that the elements in the chain are

$$
\frac{r+1}{2}=\left\{\left\{a_{\left(\frac{r+1}{2}\right) j}\right\}: \frac{r-1}{2} \leqslant m \leqslant \frac{2 e-r+3}{2}\right\}
$$

where $r<e, r$ is odd and $c$ is a positive integer.

Proof. Each chain covers two rows and two columns, namely the $i$ th column and the $(e-i+1)^{\text {th }}$ column, and for the vertical-path chain the $i$ th column and the $(e-i+1)^{\text {th }}$ column become the same, and thus we get; $i=e-i+1$ or, $i=\frac{e+1}{2}$, and similarly for the horizontal-path chain $i=\frac{r+1}{2}$. So the vertical path chain will be in column $\frac{e+1}{2}$ and will start from $i$ th column and $i$ th row and $i$ here is $\frac{e+1}{2}$ so the rows start from the $\left.\frac{e+1}{2}\right)$ th row. and now it will continue till the $(r-i+1)^{\text {th }}$ row. By putting the value of $i$ we get; $r-\frac{e+1}{2}+1=\frac{2 r-e+3}{2}$ so the last row will be $\frac{2 r-e+3}{2}$ and thus we have the chain $\frac{e+1}{2}=\left\{a_{m\left(\frac{e+1}{2}\right)}: \frac{e-1}{2} \leqslant m \leqslant \frac{2 r-e+3}{2}\right\}$ where $e<r, e$ is odd and $c$ is a positive integer. The same method is applied for the horizontal-path chain.

From Theorem 3, there are two design structures of the rectangle-path nested chains abacus. The vertical rectangle-path nested chains with $c$ vertical rectangular chains and one vertical-path chain is a nested chain abacus with $e<r$ and $e$ is odd, where $e$ and $r$ are the number of the columns and rows, respectively. Example 3.3 provides the illustration of this design structure.

\section{Crollary 1}

Let $\mathfrak{N}$ be a nested chain abacus with $e$ column, $r$ rows and $c$ rectangular chains. Then $V$ is the arithmetic sequence for the number of positions in the nested chains abacus, with -8 as the common difference of successive terms where $V_{i}$ is the number of positions in chain $i$ and $\left\langle V_{i} \gg=\ll\right.$ $V_{1}, V_{2}, V_{3}, \cdots, V_{\frac{e}{2}} \gg$ for $1 \leqslant i \leqslant c$.

Proof. Let $V_{i+1}$ and $V_{i}$ represent the number of positions in chain $i+1$ and chain $i$, respectively, where $i=1,2, \cdots, c$, where $c=\frac{e}{2}$ if the nested chain abacus is a vertical nested chain abacus and $c=\frac{r}{2}$ if the nested chain abacus is a horizontal nested chain abacus, as mentioned in Theorem 2 . Thus

\subsection{Singleton nested chain abacus}

$$
[2 r+2 e-4(2(i-1)-1)]-[2 r+2 e-4(2 i-1)]=-8 \text {. }
$$

The singleton nested chain abacus consists of rectangular chains and a singleton chain. Theorem 4 clarifies the construction of Singleton chain in nested chain abacus.

\section{Theorem 4}

Let the $r \times e$ matrix A represents bead positions and empty bead positions in the nested chain abacus with $e$ columns and $r$ rows, where $e=r$ and $e$ is odd. Then, singleton chain is a position $a_{m\left(\frac{e+1}{2}\right)}$ located in column $\frac{e+1}{2}$ and row $\frac{e+1}{2}$.

Proof. Each chain covers two rows and two columns, the $i$ th column and the $(e-i+1)^{\text {th }}$ column, and for the singleton chain the $i$ th column and the $(e-i+1)^{\text {th }}$ column become the same, and thus we obtain $i=e-i+1$ or, $i=\frac{e+1}{2}$ and similarly for the rows.

\section{Conclusion}

We have developed an algorithm for the construction of a nested chain abacus, as a representation of the previously described $n$-connected ominoes $[7,8,9]$. At this stage, two questions can be asked: - Could we use the topological stracture of nested chain abacus to classify classes for $n$-connected ominoes?

- Could we use the design structure of nested chain abacus to construct the generating function for n-connected ominoes? 


\section{References}

1. James, G., Lyle, S. and Mathas, A. 2006. Rouquier blocks. Mathematische Zeitschrift, 252(3): 511.

2. James, G. D. 1978. Some combinatorial results involving Young diagrams. In Mathematical proceedings of the cambridge philosophical society, Cambridge University Press: 83 (1): 1-10.

3. Andrews, G. E. 1998. The theory of partitions (No. 2). Cambridge University Press.

4. Loehr, N. A. 2010. Abacus proofs of Schur function identities. SIAM Journal on Discrete Mathematics, 24(4): 1356-1370.

5. Savage, C. D. 2016. The mathematics of lecture hall partitions. Journal of Combinatorial Theory, Series A, 144: 443-475.

6. Eman F Mohommed, 2019. Constructing a Nested Chain in James Abacus Diagram, Journal of Physics: Conference Series, 1294 (3): 032019

7. Golomb, S. W. 1954. Checker boards and polyominoes. The American Mathematical Monthly, 61(10): 675-682.

8. Klarner, D. A. and Rivest, R. L. 1973. A procedure for improving the upper bound for the number of n-ominoes. Canadian Journal of Mathematics, 25(3): 585-602.

9. Golomb, S. W. 1996. Polyominoes: puzzles, patterns, problems, and packings (Vol. 16). Princeton University Press. 